\title{
A LOSS FUNCTION APPROACH TO GROUP PREFERENCE AGGREGATION IN THE AHP
}

\author{
Keun-Tae Cho and Yong-Gon Cho \\ School of Systems Engineering Management, Sungkyunkwan University \\ 300 Chonchon-dong, Jangan-gu, Suwon, Kyounggi-do, 440-746, South Korea \\ ktcho@yurim.skku.ac.kr, ygjo94@iesys.skku.ac.kr
}

Keywords: AHP, consistency ratio, evaluation quality, group decision, group weight, loss function

Summary: The Analytic Hierarchy Process (AHP) is a useful method in aggregating group preference. We suggest a new method that uses consistency ratio as group evaluation quality. For this method, we introduce Taguchi's loss function. We also develop an evaluation reliability function to derive group weight. Lastly, we perform four experiments in order to confirm validity of this method.

\section{Introduction}

The AHP as a growing field in both its theoretical and applied ramifications has been applied widely in decision making. One of the topics on which research concentrates is the problem of group judgments aggregation and consistency ratio. The previous group aggregation methods keeps Saaty's rule that consistency ratio of individual pairwise comparison should be less than 0.1. That is, in aggregating individual judgments to a group opinion, one takes only individual judgments whose consistency ratio is less than 0.1. However, judgments are frequently inconsistent, and practically, pairwise comparison matrices rarely satisfy the consistency criterion. Thus these methods tend to ignore many information of evaluation by using Saaty's consistency ratio. While previous methods dealt with weight of each evaluator, there have been no studies that tried to aggregate group priority by using weight of a group unit in aggregating individual judgment. In order to overcome such problems, we introduce the concept of Taguchi's loss function and develop a loss function approach, which is a new method for improving group judgments aggregation. The loss of evaluation quality will be used as the weight of group in aggregating group judgments. We call this method the Weighted After Geometric Mean Method (WAGMM) and the Weighted After Arithmetic Mean Method (WAAMM) for convenience.

\section{Group Decision Making in the AHP}

Group decision making involves weighted aggregation of different individual preferences to obtain a single collective preference. This subject has received a great deal of attention from researchers in many disciplines. Two of the methods that have been found to be most useful are the geometric mean method and arithmetic mean method. Unfortunately, it is extremely difficult to accurately assess and quantify changing preferences, and to aggregate conflicting opinions held by diverse group.

\section{Loss Function Approach}

Loss function has three types of characteristics such as nominal-is- best characteristics, Smaller-is-better characteristics and Large-is-better characteristics.

We first introduce an expected loss derived from Taguchi's loss function in order to obtain a collective and aggregated weight of an evaluation group. We take a loss function for smaller-is-better characteristics, according to the fact that it is good judgments, as consistency ratio is near to zero. An expected loss for this characteristic is obtained by the sum of mean and variance as the following equation (1).

$$
L=k E\left(y^{2}\right)=k\left(\sigma^{2}+\mu^{2}\right)
$$


We next propose a new function called the 'evaluation reliability function', which transforms an expected loss to a collective weight of the group. The 'evaluation reliability function' is defined as the following equation (2).

$$
\begin{aligned}
& F(X)=1 \quad X=0 \\
& F(X)=\operatorname{Exp}(-\alpha X) \quad 0<X<\text { Tolerancelimit } \\
& F(X)=0 \quad X \geq \text { Tolerancelimit }
\end{aligned}
$$

Where, $\alpha$ is coefficient by each dimension. When an expected loss for consistency ratio of a group becomes 0 , the weight of the group has a value of 1 . When an expected loss is beyond tolerance limit, the group has a value of 0 .

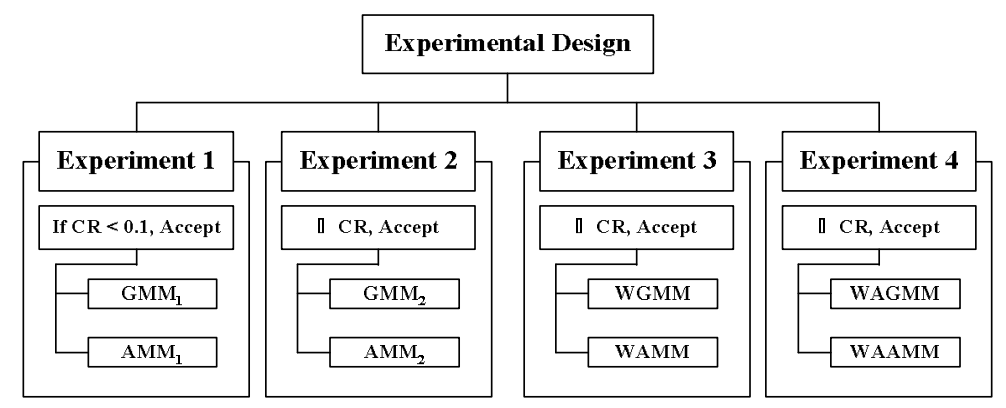


dimensions. We made an evaluation reliability function into exponential function. It is necessary to develop various form of evaluation reliability function according to characteristic of a group. 
